\section{Kidney \\ Blood Pressure \\ Research}

\title{
Does Proteinuria-Inducing Physical Activity Increase Biomarkers of Acute Kidney Injury?
}

\author{
Naushad A. Junglee ${ }^{a, b} \quad$ Andrew B. Lemmey ${ }^{a}$ Megan Burton ${ }^{a}$ Catrin Searell ${ }^{c}$

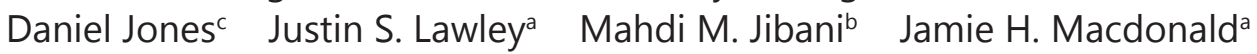 \\ ${ }^{a}$ College of Health and Behavioural Sciences, Bangor University, George Building, Bangor, Wales, LL57 \\ 2PZ, UK, bDepartment of Nephrology, Gwynedd Hospital, Betsi Cadwaladr University Health Board, \\ Penrhosgarnedd, Bangor, Wales, LL57 2PW, UK, 'Department of Biochemistry, Gwynedd Hospital, Betsi \\ Cadwaladr University Health Board, Penrhosgarnedd, Bangor, Wales, LL57 2PW, UK
}

\section{Key Words}

Acute kidney injury • Biomarkers $•$ Exercise $\cdot$ NGAL protein $•$ Proteinuria

\begin{abstract}
Aim: We sought to determine if an acute kidney injury biomarker, neutrophil gelatinaseassociated lipocalin (NGAL), would be up-regulated by high-intensity proteinuria-inducing exercise. Methods: A prospective cohort design was utilised. 100 healthy, active adults (mean age $24 \pm 4$ (SD) years) were screened for post-exercise proteinuria (PeP); 10 PeP positive and 10 PeP negative participants then completed a high-intensity exercise protocol involving an 800 meter sprint. Plasma and urinary NGAL, urinary creatinine, urinary albumin and urine volume were obtained at the following time points: pre-run, immediately post-, 25 minutes, one hour and two hours post-run. Results: Following high-intensity exercise, $64 \%$ of participants had urinary NGAL concentrations above the normal range, particularly at 25 minutes post $(P=$ 0.002). However, there was no difference in NGAL response between PeP positive and negative groups and plasma NGAL was decreased, not elevated, following exercise $(P=0.002)$. In some individuals normalizing urinary NGAL for urinary creatinine attenuated elevations. Urinary NGAL was also negatively correlated with urine volume $(r=-0.701, P=0.005)$. Conclusion: Proteinuria susceptibility did not influence an acute injury biomarker response to exercise. Nevertheless, urinary NGAL was elevated by exercise, possibly due to increased production by the proximal tubule, increased plasma clearance (given the decrease in plasma NGAL) and/ or a concentrating effect of exercise-induced oliguria. Until correct normalisation of urinary biomarkers is determined, NGAL should be interpreted cautiously in exercise and acute kidney injury-induced oliguria. The inter-individual NGAL response to exercise also warrants further investigation.
\end{abstract}




\section{Kidney Blood Pressure Research}

Kidney Blood Press Res 2012;36:278-289

\begin{tabular}{l|l}
\hline DOI: $10.1159 / 000343417$ & (C) 2012 S. Karger AG, Basel
\end{tabular}

Published online: November 28, 2012

www.karger.com/kbr

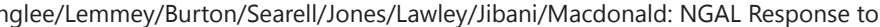
Exercise Proteinuria

\section{Introduction}

During exercise, the kidneys maintain homeostatic composition of extracellular fluid, thus enabling regulation of vascular volume and preservation of cardiac output. However, high-intensity physical activity is associated with marked perturbances in renal function [1]. In fact, up to a 30 to $40 \%$ decrease in renal blood flow has been observed as blood is diverted to other metabolically demanding tissues such as the active musculature. In addition, enhanced proteinuria and hematuria, reductions in glomerular filtration rate (GFR), and impaired handling of electrolytes and amino acids are also apparent [2].

When chronic, such perturbances are usually associated with renal pathology [3]. However, in exercise models these changes are interpreted as harmless as they typically resolve within 24 to 72 hours [4]. Nevertheless, such derangements can indicate and progress to more serious acute renal pathology. For example, in U.S. military members, acute kidney injury (AKI) has been recognized as a significant threat during physical exertion, particularly under heat stress [5]. A delay in diagnosis of AKI is not uncommon and hemodialysis may be required which carries significant morbidity.

One of the most commonly observed disturbances to kidney function with exercise is post-exercise proteinuria (PeP). Several mechanisms have been proposed for this condition but PeP shows large inter- and intra-individual variation $[1,6]$. Why such variability exists in renal responses to exercise and why and some individuals progress to more serious renal pathology remains unclear. Until recently, one obstacle has been a lack of sensitive biomarkers of kidney injury. Serum or plasma creatinine $(\mathrm{Cr})$ is the most widely utilized measure of renal function. At present, it is essential for the diagnosis of AKI and has been repeatedly used to demonstrate the declines in renal function during exercise [1, 7]. Despite its convenience, $\mathrm{Cr}$ is not a kidney injury biomarker per se and has its deficiencies such as a delayed response in AKI [8]. Thus, there has been avid interest in identifying biomarkers which are capable of detecting kidney injury (and therefore impaired renal function) much earlier than blood Cr. One promising biomarker is neutrophil gelatinase-associated lipocalin (NGAL) [9]. However, the behaviour of these biomarkers to exercise has only recently begun to be investigated [10]. Responses to exercise protocols of varying intensity and duration remain unknown. Whether individuals who are susceptible to PeP also experience greater up-regulation in NGAL due to nephron-related stress has also not been determined.

Given the physiological aberrations to kidney function known to occur during exercise, their unexplained inter-individual response and the recent development of more sensitive biomarkers of kidney injury, the aim of this experiment was to determine if NGAL is up-regulated following a short duration high-intensity bout of proteinuria-inducing exercise (800 meter sprint). In this model, we hypothesized that: i) NGAL would show a significant but transient rise above normal ranges (but not approaching levels close to those seen in AKI); and ii) the elevations would be greater in those with PeP compared to those without. If supported, this would reveal further insights into site-specific renal stresses during exercise and provide a novel and more sensitive method of assessing the acute effects of exercise upon renal function. Additionally, any elevations in NGAL post-exercise may have implications in biomarker interpretation within the clinical environment.

\section{Materials and Methods}

\section{Study design and participants}

We employed an observational prospective cohort design (Fig. 1). In the first stage of the study, 100 potential participants (mean age $( \pm$ SD) $=24( \pm 4)$ years; 48 female; 52 male) were approached via university and local sports clubs between September 2010 and July 2011 and were screened for presence of PeP. Subsequently, $10 \mathrm{PeP}$ positive and $10 \mathrm{PeP}$ negative participants, who were conveniently sampled and not purposefully matched, completed the second stage of the study, which involved collection of blood and urine samples following an experimental exercise protocol designed to induce proteinuria. All 


\section{Kidney Blood Pressure Research}

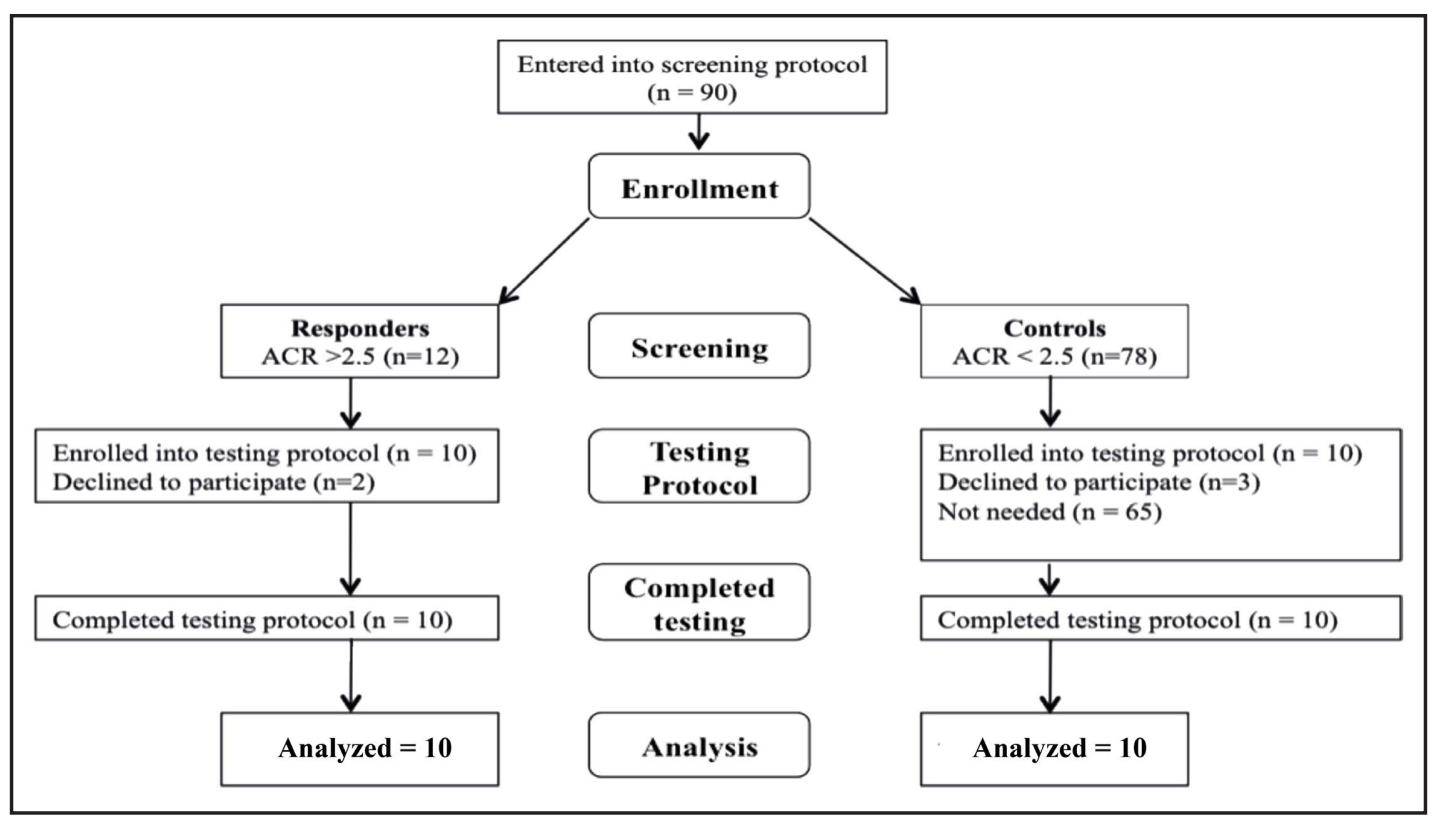

Fig. 1. Study design flowchart.

participants provided written informed consent and ethical approval was provided by the College of Health and Behavioural Sciences, Bangor University ethics committee.

\section{Screening protocol for exercise proteinuria}

All participants completed a brief health questionnaire, which showed that none had significant medical conditions or took medications that could potentially influence kidney function (e.g. non-steroidal anti-inflammatory drugs). Screening for PeP was conducted through the following methods: rested individuals (i.e. who had not performed strenuous exercise in the last 72 hours) were asked to provide a urine sample prior to the start of their normal exercise routine. Approximately 30 minutes following this exercise a further urine sample was taken. Both samples were stored in ice boxes during transportation and auto-analysed for albumin:creatinine ratio (A:Cr) estimation within 24 hours of collection (both by Olympus AU2700 analyser; creatinine reagent OSR6178, Beckman Coulter, California, USA (kinetic Jaffe method); Microalbumin reagent MA2426, Randox Laboratories, Crumlin, UK). Estimated glomerular filtration rate was also calculated by the CKD-EPI (Chronic Kidney Disease Epidemiology Collaboration) equation [11].

There are no clear guidelines to the acceptable A:Cr limits for post-exercise proteinuria in healthy subjects, although various literature does exist regarding provocation of PeP in diabetics as part of a risk assessment of future diabetic nephropathy [12]. Therefore, to define whether an individual was PeP positive the well-established criteria for significant microalbuminuria in diabetics was adopted and applied to the A:Cr difference between pre to post-exercise. Thus, subjects were defined as PeP positive if their A:Cr rise was greater than $2.5 \mathrm{mg} \cdot \mathrm{mmol}^{-1}$ (male) or $3.5 \mathrm{mg} \mathrm{mmol}^{-1}$ (female) [13]. Those not fulfilling such increases were deemed PeP negative.

\section{Experimental protocol for proteinuria-inducing exercise}

All PeP positive and randomly selected PeP negative subjects were invited to complete the full experimental protocol. Eventually twenty participants (Fig. 1) completed the protocol, performing an 800 meter sprint at the University athletic track between September 2010 and August 2011. This form of high intensity, lactate-generating exercise is recognised as an optimal protocol for inducing PeP [14]. A day prior to any sampling of urine and blood, all participants were required to drink two liters of water (in addition to normal fluid intake) to ensure adequate hydration. Hydration status was assessed by using a portable meter (Pocket Pal-Osmocheck, Vitech Scientific, Partridge Green, UK) to assess urine osmolality immediately prior to completing the experimental protocol. If the value exceeded $700 \mathrm{mOsmols} \cdot \mathrm{KgH}_{2} 0^{-1}$, individuals 


\section{Kidney \\ Blood Pressure Research}

Kidney Blood Press Res 2012;36:278-289

\begin{tabular}{l|l}
\hline DOI: $10.1159 / 000343417$ & (C) 2012 S. Karger AG, Basel
\end{tabular}

Published online: November 28, 2012

www.karger.com/kbr

nglee/Lemmey/Burton/Searell/Jones/Lawley/Jibani/Macdonald: NGAL Response to Exercise Proteinuria

were asked to re-attend when adequately hydrated [15]. Participants were also asked to refrain from heavy exercise for at least four days and not to eat during the two hours before testing.

Subjects ran in groups of two or three to encourage competition and to elicit maximal effort. As a measure of exercise intensity (anaerobic energy production) during exercise, blood lactate was measured pre- and immediately post-run (Lactate Pro Lt-1710, Arkray, Kyoto, Japan) [16]. Following the run, participants were rested. Venous blood and urine specimens were collected prior to, immediately post(0 minutes), 25 minutes post-, one hour post- and two hour post-run. These time points were selected to maximise the chances of early detection of PeP and NGAL, as elevations in PeP have been shown to be maximal 20-30 minutes after exercise, while NGAL increases have been confirmed as early as two hours after the onset of AKI $[1,9]$. Urine volume was also recorded at each time point. Hemoglobin $(\beta$-Hemoglobin Hemocue AB photometer, Hemocue Ltd, Dronfield, UK) and hematocrit (Hawksley and sons Ltd, Sussex, UK) were also measured for subsequent plasma volume shift calculations as described by Dill and Costill [17]. Following this, all blood specimens were centrifuged; plasma and urine aliquots were stored at $-80^{\circ} \mathrm{C}$ for subsequent biochemical analysis.

Ascertainment of albumin:creatinine and urine and plasma NGAL

A:Cr assessments were performed as described above. NGAL concentrations were determined using a commercially available enzyme-linked immunosorbent assay (NGAL Rapid ELISA, Bioporto, Gentofte, Denmark). Absorbances were read at a wavelength of $450 \mathrm{~nm}$ by a microplate reader (Opsys MR, Dynex Technologies, Worthing, UK). The normal ranges quoted by the kit manufacturer for urinary NGAL (UNGAL) and plasma NGAL (pNGAL) concentrations were 0.7-9.8 ng.ml-1 and 37-106 ng.ml-1, respectively [18]. According to the kit insert, intra-assay variation was determined by measurement of NGAL in two urine and two plasma samples with eight replicates each. The CVs for both urine samples were 3.4\% and 4.3\%, respectively, and for plasma, $2.9 \%$ and $1.9 \%$. Inter-assay variation was calculated through a similar process with two dilute urine and two dilute EDTA plasma samples in two replicates. The CVs for both urine samples were $4.7 \%$ and $22.7 \%$, respectively, and for plasma, $11.4 \%$ and $12.4 \%$.

As with A:Cr, UNGAL concentrations were corrected for urine creatinine to account for changes in urinary concentration or dilution. Therefore, creatinine corrected UNGAL (UNGAL:Cr) was expressed as ng NGAL / mmol urine creatinine $\left(\mathrm{x} 10^{-3}\right)$. Additionally, pNGAL concentrations were corrected for plasma volume change [17].

\section{Statistical analysis}

SPSS version 18.0 (IBM, Chicago, IL) was used for all statistical analysis. Statistical significance was accepted at $P \leq 0.05$. Data for all dependent variables were examined for normality through visual inspection of histogram plots and Shapiro-Wilk tests. In the case of descriptive data and pNGAL, mean $( \pm S D)$ are stated. However urine volume, A:Cr, UNGAL and UNGAL:Cr were not normally distributed and remained markedly skewed despite attempts at data transformation. Thus, these non-parametric data are presented as median $\left(25^{\text {th }}\right.$ percentile, $75^{\text {th }}$ percentile $)$. Nonetheless, in the interests of consistency, all results were graphically presented as boxplots.

Descriptive data were examined by Student's $t$-test. Correlations between variables were determined using Pearson's $(r)$ or Spearman's rank $(\rho)$ correlation coefficient, as appropriate. For pNGAL, to examine the interaction between proteinuria group (PeP positive or PeP negative) and time (pre, post $0 \mathrm{hr}, 25 \mathrm{~min}-$, $1 \mathrm{hr}-$, and 2 hrs- post) a parametric two-way repeated measures analysis of variance was used with post hoc Tukey's tests.

For urine volume, A:Cr, uNGAL and UNGAL:Cr a Friedman's test (a non-parametric alternative to repeated measures analysis of variance) was performed to examine the effect of time. The main effects of proteinuria group and the interaction between proteinuria group and time were examined using a two-way non-parametric analysis of covariance as outlined by Quade [19]. Significant differences were examined further using post hoc Wilcoxon Signed-Ranks with Bonferroni corrections for multiple comparisons.

For the primary outcome measure of UNGAL, a power calculation revealed that six participants were needed per group to detect a smallest important change in means of $4.6 \mathrm{ng} \cdot \mathrm{ml}^{-1}$ (the increase required to elevate uNGAL above the normal range), assuming a within subject error of $1.7 \mathrm{ng} . \mathrm{ml}^{-1}$, and allowing for a 5 and $20 \%$ chance of making a Type I or Type II error, respectively [20]. 


\section{Kidney \\ Blood Pressure Research}

Fig. 2. Effect of an 800 meter run on urine volume. Data are medians (thick lines) and interquartile ranges (boxes). Open circles and asterixes are outliers. There was a significant effect of time $(P=$ 0.001 by Friedman's test). \#, difference compared to preexercise sample point by post hoc test.

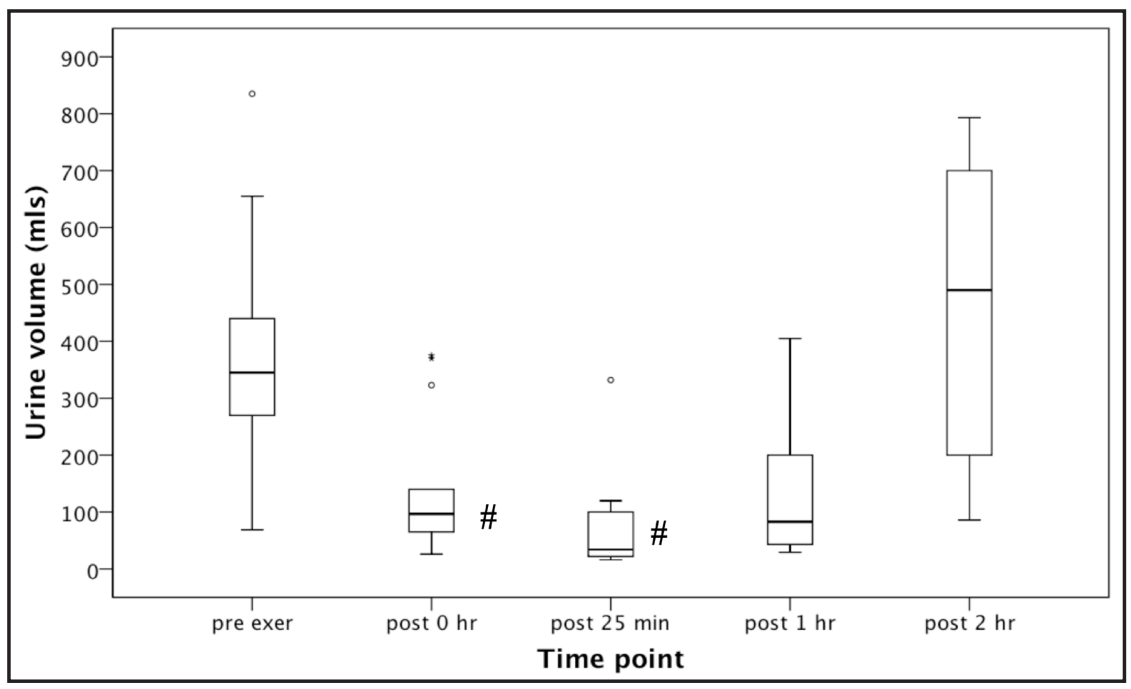

Fig. 3. (a) Effect of an 800 meter run on albumin:creatinine ratio in nonmacro and macrop r o t e i n u r i a subgroups. Data are medians (thick lines) and in te rquartile ranges (boxes). Open circles are outliers. There was a significant i n t e r a c t i o n between time and macroproteinuria status $(P=0.001$ by Quade's test). \#, difference between nonmacro and macroproteinuria groups by post hoc test. (b) Individual plots for albumin:creatinine ratio following an 800 meter run.

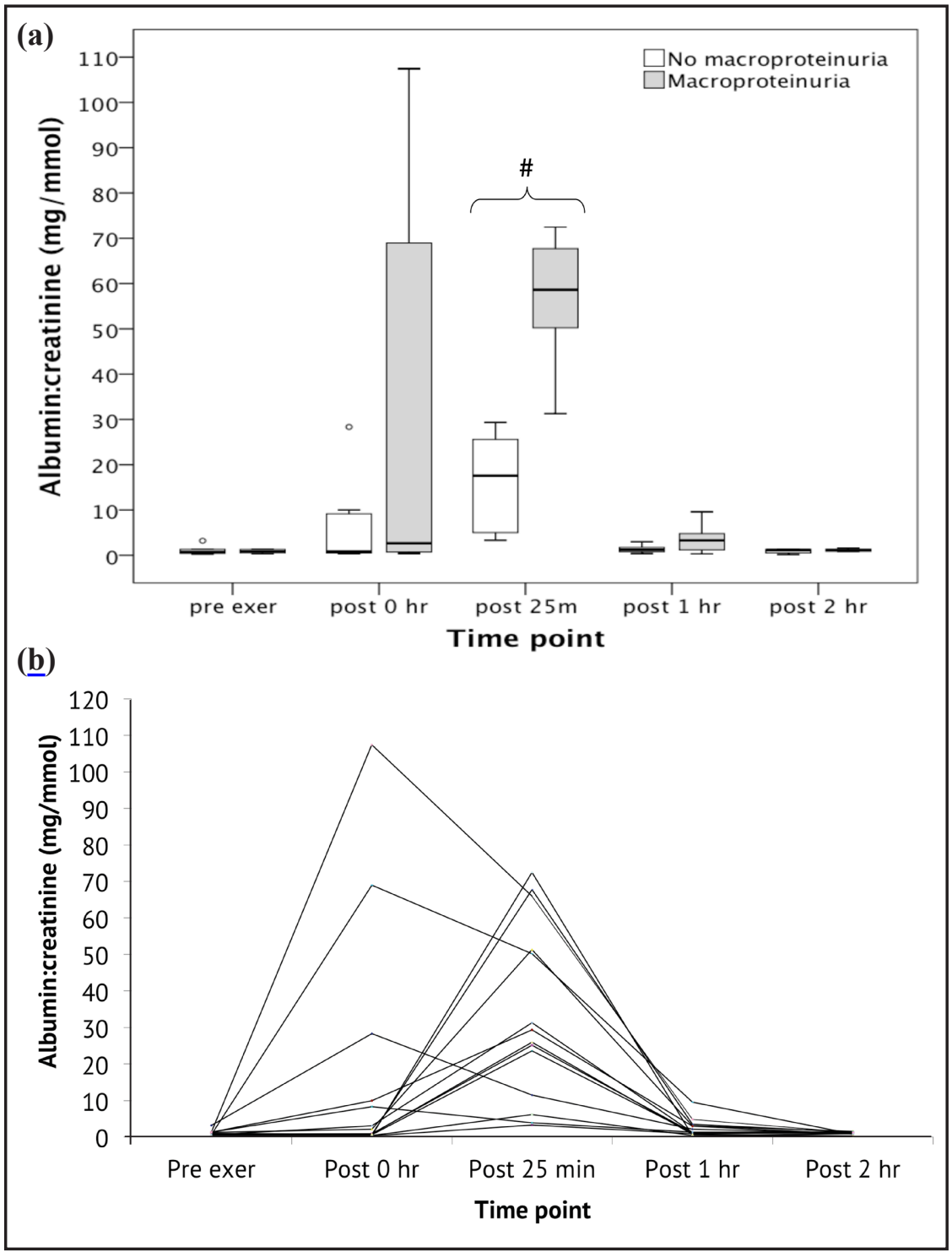




\section{Kidney Blood Pressure Research}

Kidney Blood Press Res 2012;36:278-289

\begin{tabular}{l|l}
\hline DOI: $10.1159 / 000343417$ & (C) 2012 S. Karger AG, Basel
\end{tabular}

Published online: November 28, 2012

www.karger.com/kbr

\section{Results}

\section{Descriptive data}

All participants were Caucasian. Estimated glomerular filtration rate was similar in both groups (PEP +: $121( \pm 8)$; PEP -: $\left.115( \pm 13) \mathrm{ml} \cdot \mathrm{min}^{-1} \cdot 1.73 \mathrm{~m}^{2-1} ; P=0.3\right)$ and within healthy ranges for all participants. In the PeP positive group age was slightly higher (PEP +: $24( \pm 5)$; PEP -: $20( \pm 2)$ yrs; $P=0.04)$ and there were more females (PEP +: 5 females; PEP -: 2 females).

As there were no differences between groups for hydration status and exercise intensity by independent $t$-tests, data from both groups were combined. Pre-run urine osmolality was $173( \pm 119) \mathrm{mOsmols} \cdot \mathrm{KgH}_{2} \mathrm{O}^{-1}$, indicating good hydration status prior to testing. There was the expected elevation of serum lactate between pre- and 0 hours post-run $(1.2( \pm 0.4)$ and $8.3( \pm 3.1) \mathrm{mmol} \cdot \mathrm{l}^{-1}$, respectively; $\left.P<0.001\right)$, indicating physical exercise was completed at a high intensity.

\section{Urine volume}

Only $14 / 20$ participants $(70 \%)$ were able to provide urine samples at all time points following the 800 meter run. Thus, subsequent reporting of results and statistics refers only to this cohort. This observation was accounted for by an exercise induced anti-diuretic effect that was most pronounced at 25 minutes, when only $38(28,99) \mathrm{ml}$ of urine could be produced. This oliguria recovered by two hours to $420(204,586) \mathrm{ml}$. Overall, there was a significant effect of time $(P=0.001$; post-hoc analyses, pre to 0 hours: $P=0.004$; pre to 25 minutes: $P=0.001$; pre to two hours $P=0.5$; Fig. 2 ). Response in urine volume was similar between macroproteinuria and non-macroproteinuria groups (no interaction effect: $P=$ $0.5)$; therefore both groups are combined in Fig. 2.

\section{Urinary albumin: Creatinine}

Despite identification of PeP positive and PeP negative subjects during screening, the experimental protocol yielded a rise in A:Cr post-exercise in all participants who provided urine samples by 25 minutes. At this time point, mean A:Cr peaked at $27.6(11.7,50.5)$ $\mathrm{mg} \cdot \mathrm{mmol}^{-1}$ before returning close to pre-exercise levels of $1.2(0.9,1.4) \mathrm{mg} \cdot \mathrm{mmol}^{-1}$, by two hours with a significant effect of time $(P<0.001$; post-hoc analyses, pre to 25 minutes: $P$ $=0.001$; pre to two hours: $P=0.4$ ). There was a trend for a positive correlation between percentage change in serum lactate and percentage change in A: $\operatorname{Cr}$ (between pre and 25 minutes; $r=0.493 ; P=0.07$ ).

As all participants unexpectedly developed PeP at 25 minutes, a new A:Cr cut-off criteria was applied to examine the quantitative effects of proteinuria upon NGAL release. Therefore, the participants were divided into macroproteinuria $\left(\mathrm{A}: \mathrm{Cr}>30 \mathrm{mg} \cdot \mathrm{mmol}^{-1} ; \mathrm{N}=6\right)$ and non-macroproteinuria (A:Cr $<30 \mathrm{mg} \cdot \mathrm{mmol}^{-1} ; \mathrm{N}=8$ ) groups by assessing their peak $\mathrm{A}: \mathrm{Cr}$ value at 25 minutes [1]. Baseline eGFR and age were not significantly different between these two newly defined groups (data not shown). Consequently and by design, for A: $\mathrm{Cr}$ there was a time $\times$ macroproteinuria status interaction (Fig. 3a). Examination of individual plots clearly showed that most peaks in A:Cr occurred at 25 minutes, but there were five individuals who peaked earlier immediately post-exercise (Fig. 3b).

\section{Urinary NGAL}

There were increases in UNGAL following exercise which peaked at $12.8(6.2,23.2)$ $\mathrm{ng} \cdot \mathrm{ml}^{-1}$ at 25 minutes before decreasing below baseline levels to $0.8(0.02,3.3) \mathrm{ng} \cdot \mathrm{ml}^{-1}$ at two hours (significant effect of time: $P=0$. 002; post-hoc analyses, pre to 25 minutes: $P=0.04$ (not significant after Bonferroni correction); pre to two hours post: $P=0.004$; Fig. 4a). At 25 minutes and two hours, uNGAL was negatively correlated with percentage change in urine volume (pre to 25 minutes; $\rho=-0.701 ; P=0.005 ; 25$ minutes to two hours; $\rho=-0.552 ; P=$ $0.04)$. Response in uNGAL was similar between macroproteinuria and non-macroproteinuria groups (no interaction effect; $P=0.6$ ); therefore both groups are combined in Fig. 4a. Individual data plots suggested a cluster of uNGAL peaks occurring at 0 and 25 minutes post 


\section{Kidney \\ Blood Pressure Research}

Fig. 4. (a) Effect of an 800 meter run on urinary NGAL concentration. Data are medians (thick lines) and interquartile ranges (boxes). Open circles and asterixes are outliers. Dotted line indicates upper limit of normal range for urinary NGAL. There was a significant effect of time $(P=0.002$ by Friedman's test). \#, difference compared to preexercise sample point by post hoc test; $¥$, difference compared to pre - e x e r c is e sample point by post hoc test but not significant after Bonferroni correction. Individual plots for urinary NGAL concentration following an 800

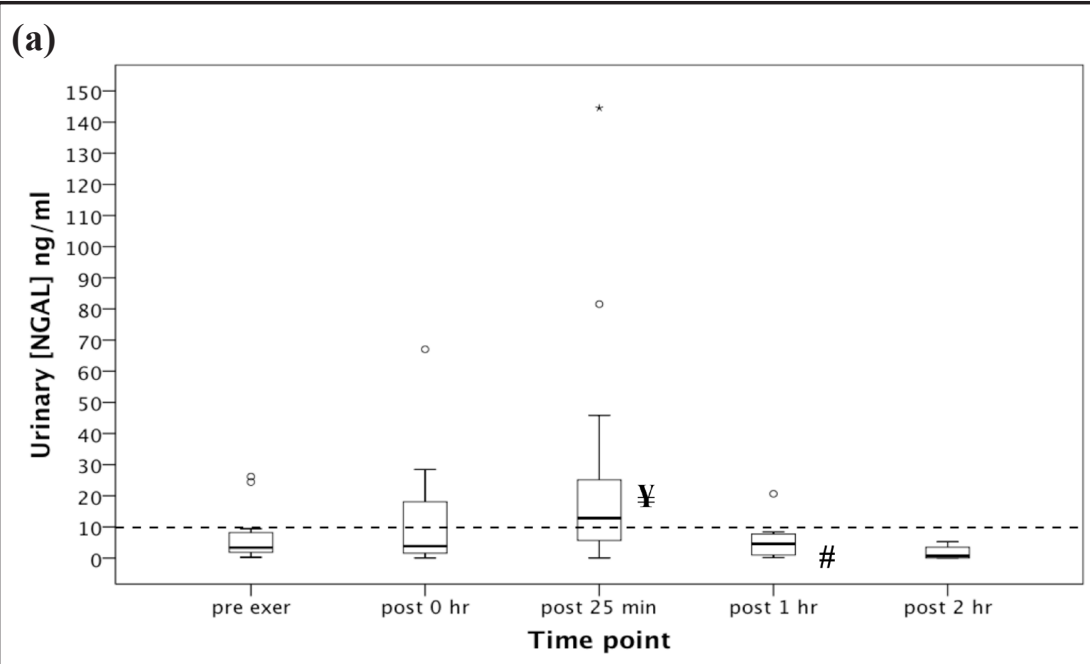

(b)

Junglee/Lemmey/Burton/Searell/Jones/Lawley/Jibani/Macdonald: NGAL Response to Exercise Proteinuria

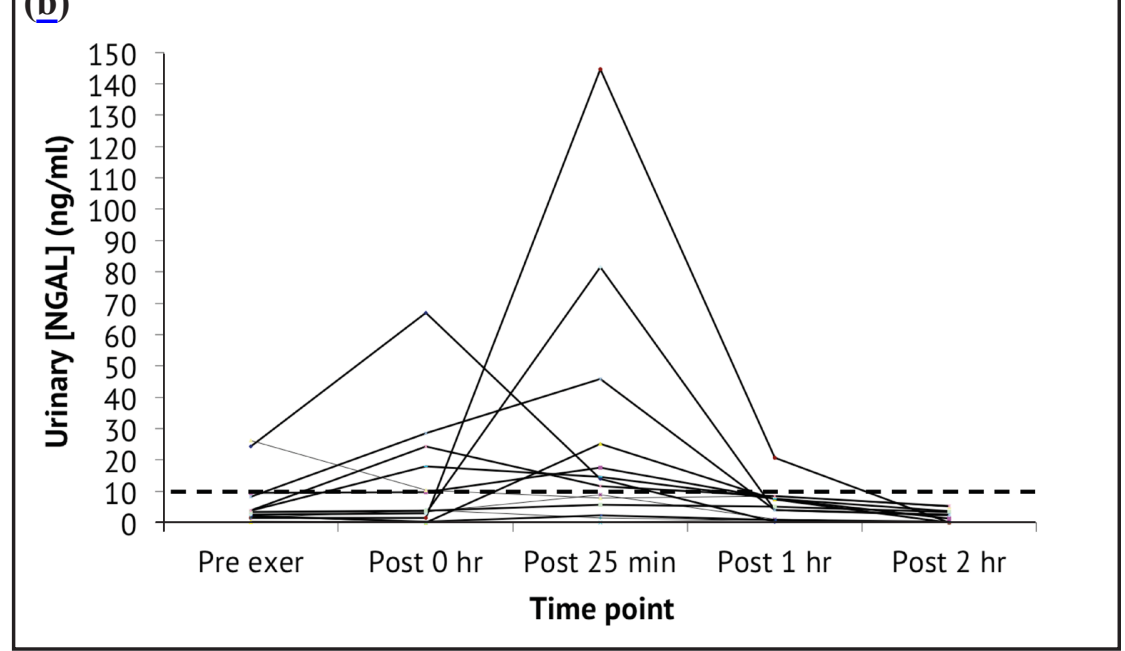
meter run.

although there was marked inter-individual variation (Fig. 4b). Throughout the entire postexercise period, nine individuals (64\%) showed an elevation above the normal range at one or more time points - indeed one subject obtained a concentration of $145 \mathrm{ng} \cdot \mathrm{ml}^{-1}$ (Fig. $4 \mathrm{~b}$ ).

Urinary NGAL: Creatinine

Although median uNGAL:Cr appeared to be relatively stable at pre, 0 hours and 25 minutes, it fell significantly at two hours to $0.42 \times 10^{-3}\left(0.02 \times 10^{-3}, 0.1 \times 10^{-3}\right) \mathrm{ng} \cdot \mathrm{mmol}^{-1}$ with a significant effect of time $(P=0.002$; post-hoc analyses, pre to two hours: $P=0.004$; Fig. 5a). Response of uNGAL:Cr was similar between macro- and non-macroproteinuria groups (no interaction effect; $P=0.241$ ); therefore both groups are combined in Fig. 5 . When individual data were inspected, there were clusters of UNGAL:Cr peaks occurring at 0 and 25 minutes although there was marked inter-individual variation (Fig. 5b).

\section{Plasma NGAL}

Baseline pNGAL was $73.0( \pm 17.0) \mathrm{ng} \cdot \mathrm{ml}^{-1}$ and there was a significant effect of time $(P$ $=0.002$ ). However this was due to a decrease in pNGAL concentration from baseline at 25 minutes to $68.8( \pm 15.0) \mathrm{ng} \cdot \mathrm{ml}^{-1}$ and at one hour to $64.5( \pm 16.7) \mathrm{ng} \cdot \mathrm{ml}^{-1}$ (post-hoc analyses, pre and 25 minutes: $P<0.001$; pre and one hour: $P=0.007$; pre and two hours: $P=0.03$ (not significant after Bonferroni correction); Fig. 6). Similar to uNGAL and uNGAL:Cr, there was 


\section{Kidney \\ Blood Pressure Research}

Fig. 5. (a) Effect of an 800 meter run upon urinary NGAL:creatinine ratio. Data are medians (thick lines) and in terquartile ranges (boxes). Open circles and asterixes are outliers. There was a significant effect of time $(P=0.002)$. $\#$, difference compared to preexercise sample point by post hoc test. (b) Individual plots for urinary NGAL:creatinine ratio following an 800 meter run.
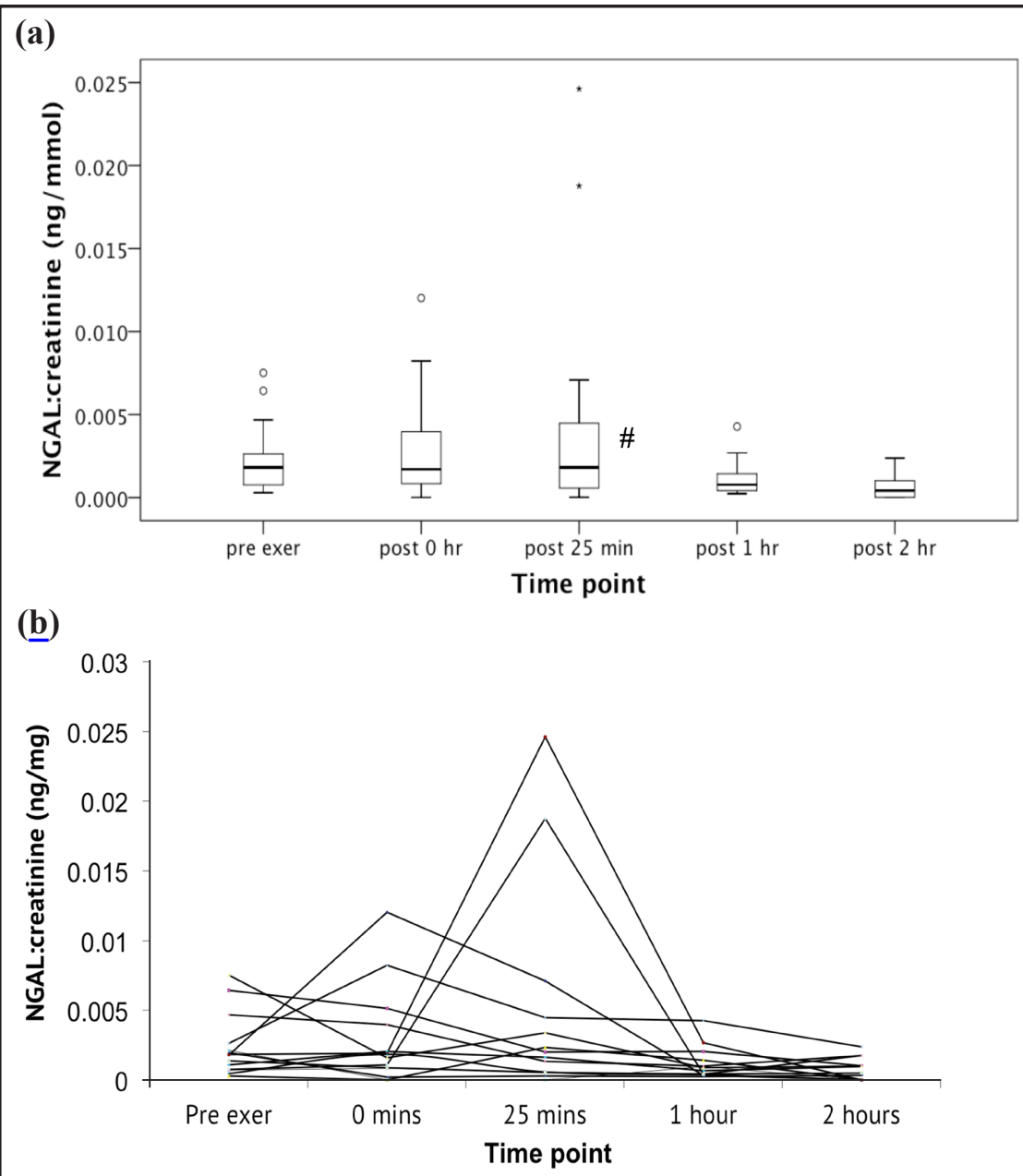

no interaction with type of proteinuria for pNGAL $(P=0.7)$. Throughout the entire postexercise period, no individual went above or below the normal range for pNGAL at any time.

\section{Discussion}

To our knowledge, this is the first study to examine NGAL kinetics and its association with proteinuria following short duration high-intensity exercise. These data support our initial hypothesis that urinary NGAL would show a significant but transient rise following an acute bout of high-intensity exercise. However, this response was not uniform and demonstrated large inter-individual variation. Furthermore, there was a negative correlation between UNGAL and percent change in urine volume, and median increases in UNGAL were attenuated when expressed relative to urinary creatinine. Taken together these data suggest that elevations in uNGAL following exercise may in part be due to a concentration effect. In addition, statistically significant decreases in plasma NGAL (pNGAL) occurred from 25 minutes post-exercise. Also the current data do not support the hypothesized association between NGAL response and the extent of post-exercise proteinuria. Nevertheless, these data provide important information on the site-specific renal stress experienced during high-intensity exercise, and have implications for interpretation of NGAL within the clinical environment. 


\section{Kidney \\ Blood Pressure \\ Research}

Kidney Blood Press Res 2012;36:278-289

\begin{tabular}{l|l}
\hline DOI: $10.1159 / 000343417$ & (c) 2012 S. Karger AG, Basel
\end{tabular}

Published online: November 28, 2012

www.karger.com/kbr

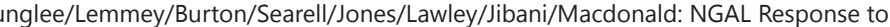
Exercise Proteinuria

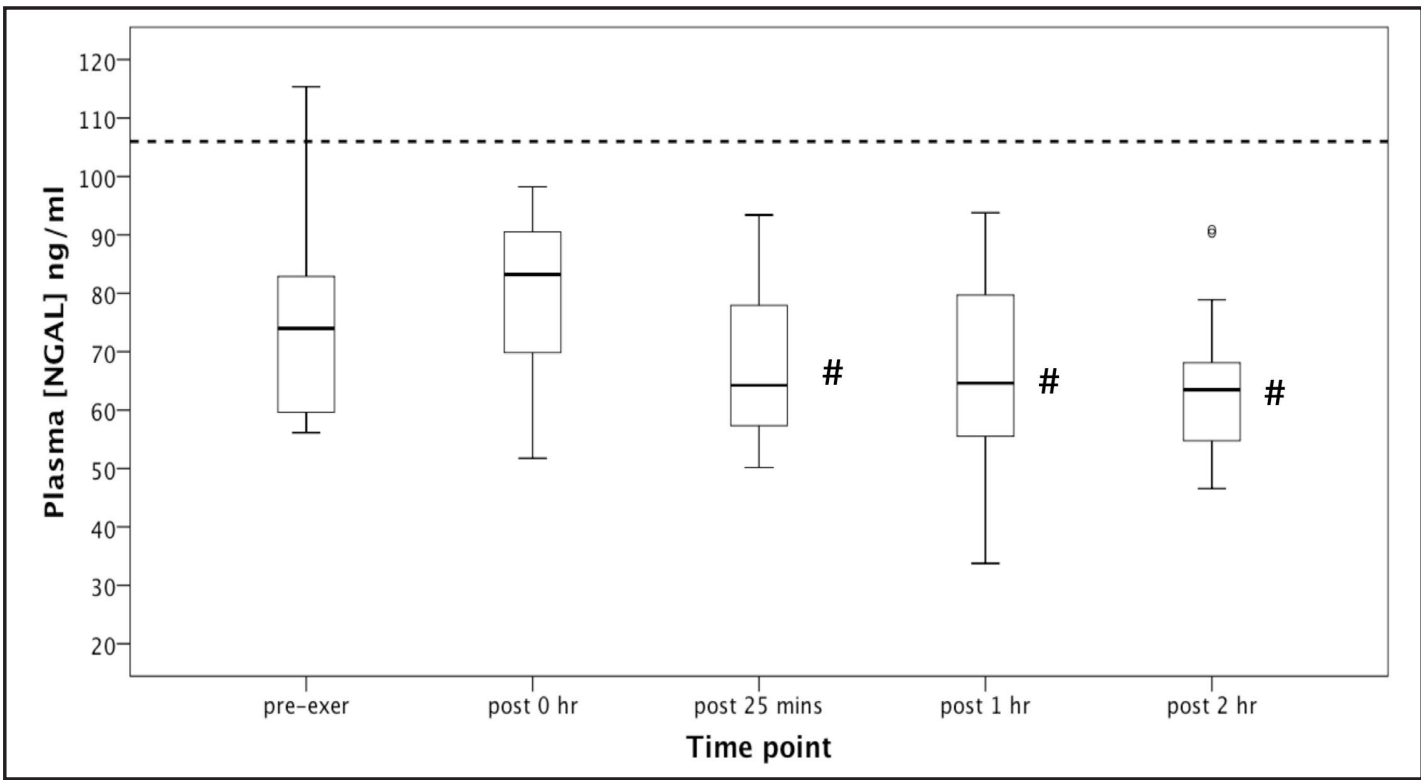

Fig. 6. Effect of 800 meter run upon plasma NGAL level over time. For the sake of consistency, data are presented as boxplots whereas data in results section are mean $( \pm S D)$. Data are medians (thick lines) and interquartile ranges (boxes). Open circles are outliers. Dotted line indicates upper limit of normal range for urinary NGAL. There was a significant effect of time $(P<0.002)$. \#, difference compared to pre-exercise sample point by post hoc test.

Although the complete functions of NGAL have yet to be elucidated, there is an early and dramatic upregulation of NGAL in AKI [9, 21-23]. More than half of participants in the current study had uNGAL elevations above the normal range at 25 minutes post-exercise, but unsurprisingly none reached the currently used cut-off diagnostic for AKI (350ng. $\mathrm{ml}^{-1}$ ) [18]. However, this cut-off has been derived from a diseased population and whether more modest elevations indicate a milder form of kidney stress or injury has not been investigated.

To date, there has previously been only one published study which reported the effect of exercise upon NGAL kinetics. In that investigation, McCullough et al. measured uNGAL in 25 individuals during and after a marathon [10]. These subjects demonstrated a significant mean elevation, from 8 (4) $\mathrm{ng} \cdot \mathrm{ml}^{-1}$ pre-marathon to 47 (29) $\mathrm{ng} \cdot \mathrm{ml}^{-1}$ immediately post marathon. The peak median uNGAL value observed in this investigation was considerably lower at 13 (IQR 17) ng. $\mathrm{ml}^{-1}$. Thus, if group averages are compared, prolonged exercise likely results in greater NGAL release compared to short-duration higher-intensity activity. However, similar to the current findings, McCullough et al. also noted wide variation for peak uNGAL with values in some individuals above $100 \mathrm{ng} \cdot \mathrm{ml}^{-1}$. Such uNGAL elevations could be a result of increased inflammatory injury to nephron units - specifically at the proximal tubule - with subsequent impairment in function [9, 24-26]. Specifically, this may affect tubular reabsorption of NGAL as reabsorption of other small molecular weight proteins such as lysozyme and $\beta_{2}-$ microglobulin are known to be inhibited by exercise $[1,27,28]$. Of note, leucocytouria has been discovered as a source of uNGAL elevations in the absence of AKI (e.g. in urinary tract infection) [29]. Detection of dimeric uNGAL isoforms from neutrophils may distinguish release of uNGAL from tubular epithelium which is predominantly monomeric [30]. In the present study, we did not collect urine samples for leucocyte quantification and the ELISA kit utilized in this study only detected monomeric uNGAL. Study of urinary sediments following exercise has demonstrated that long duration activity is more likely to result in an increased leucocytouria (and haematuria), but this finding is not universal [1]. The possible contribution from this source merits further investigation, but our urinary data suggest that even short exercise bouts induce a mild kidney stress. 


\section{Kidney Blood Pressure Research}

Kidney Blood Press Res 2012;36:278-289

\begin{tabular}{l|l}
\hline DOI: $10.1159 / 000343417$ & (C) 2012 S. Karger AG, Basel
\end{tabular}

Published online: November 28, 2012

www.karger.com/kbr

nglee/Lemmey/Burton/Searell/Jones/Lawley/Jibani/Macdonald: NGAL Response to Exercise Proteinuria

The present findings also highlight the difficulties of interpreting urinary biomarkers. Currently, there is no clear agreement on how renal biomarkers values from urine should be expressed and the assay kit utilized in this study bases interpretation of urinary results on raw values which fail to consider specific correction factors [8]. For example, the elevation in median uNGAL at 25 minutes may in part be explained by the oliguria at that same time point, a conclusion supported by the significant negative correlation with percentage change in urine volume between pre and 25 minutes. Exercise is known to exert an anti-diuretic effect with numerous studies demonstrating a decrease in free water clearance in both dehydrated and hyperhydrated states $[1,28]$. It is also plausible that the significant decrease in median uNGAL at two hours is due to relative polyuria - again supported by correlation with percentage change in urine volume. This situation is analogous to the shortcomings of measuring AKI biomarkers in urine during oliguric AKI [8]. Therefore, in an attempt to circumvent the issues surrounding changes in urinary concentration, we normalized uNGAL concentrations to urinary creatinine. Although this correction resulted in the dissipation of median NGAL rises and correlations with changes in urinary volume, it is noteworthy that some individuals continued to show substantial rises in urinary NGAL:Cr (particularly at 25 minutes).

As creatinine generation is not constant during exercise, normalisation of uNGAL to urinary creatinine could also be considered inappropriate. However, it is interesting to note that previous studies have utilised $\mathrm{A}: \mathrm{Cr}$ to demonstrate post-exercise proteinuria. Instead, observed urinary flow rate could be normalised to uNGAL concentrations, hence permitting determination of a biomarker excretion rate [8]. Unfortunately flow rates could not be calculated in the present study. As the first urine collection was done at pre-exercise and time the subject had micturated prior was not recorded, a baseline urinary NGAL flow rate could not be calculated. Of course, flow rates could be calculated subsequent to this but we feel these would be uninterpretable without a baseline. There has also been suggestion that urine osmolality and conductivity could be used as an alternative normalization measures [31]. Assuming a steady state, it could be useful. However, in short-term acute exercise of moderate intensity there is an increase in urine osmolality of around $40 \mathrm{mOsmols} . \mathrm{KgH}_{2} \mathrm{O}^{-1}$ [32]. In addition, high-intensity exercise as in our protocol may result in further decreases in tubular function. Thus, we are not confident that in a dynamic state such as exercise (or indeed AKI), these normalisation techniques would be helpful. Nevertheless, as normalisation of urinary NGAL remains problematic, this is an area that merits further investigation. Overall, the current data suggest that elevations in UNGAL should be interpreted with caution in both research and clinical settings, as elevated NGAL may in part be due to urinary concentration.

Measurement of pNGAL largely avoids the confounding factors affecting interpretation of its urinary equivalent. After correcting for plasma volume shifts, the current study showed no significant elevation in pNGAL. In contrary there was a minor yet statistically significant decline in pNGAL from 25 minutes that coincided with the peak uNGAL concentration. Thus, it is plausible that uNGAL elevations may arise from the plasma due to increased clearance into the Bowman's capsule via the glomerular capillaries. This may involve exercise-related reductions in renal blood flow to catecholamine surges with corresponding falls in GFR and increases in filtration fraction $[1,2]$.

The present study did not demonstrate any relationship between NGAL and the degree of PeP, nor between NGAL and any descriptive characteristics. Thus participants with postexercise macro-proteinuria reassuringly appear to be no more susceptible to exercise-induced kidney stress or injury than those without macro-proteinuria. Admittedly, with our small sample size a type II statistical error is possible. However the study was powered to detect a minimally important clinical change (NGAL elevations above the normal range). Additionally, distinct mechanisms seem to be responsible for post-exercise macro-proteinuria and NGAL up-regulation in nephron units. It is of note that $100 \%$ of individuals tested in the current study presented with PeP (as initially defined) following maximal exercise, supporting previous findings that when exercise is of sufficient intensity, all individuals will have some degree of PeP $[27,28]$. 


\section{Kidney \\ Blood Pressure Research}

Kidney Blood Press Res 2012;36:278-289

\begin{tabular}{l|l}
\hline DOI: $10.1159 / 000343417$ & C 2012 S. Karger AG, Basel
\end{tabular}

Published online: November 28, 2012

www.karger.com/kbr

Junglee/Lemmey/Burton/Searell/Jones/Lawley/Jibani/Macdonald: NGAL Response to Exercise Proteinuria

Limitations of this study include a lack of bladder ultrasound or urinary catherization. Although double voiding was encouraged, gold standard measurements of urine volume and therefore urine flow were not made, meaning biomarker production rate could not be calculated. To mitigate this limitation, participants arrived well hydrated, urinary data were presented relative to creatinine concentration, and NGAL was also obtained as a plasma concentration, expressed relative to plasma volume shifts. Study strengths include the use of a "real-world" exercise protocol and selection of non-elite athletes; both of which support the applicability of our results to a young active healthy population. The repeated measures design and the conservative approach to statistical analyses, using non-parametric analyses on data which is characteristically skewed, is also well-suited to our small cohort.

\section{Conclusion}

For the first time we have demonstrated that an acute bout of high intensity exercise results in transient uNGAL elevations that are independent of proteinuria. Sources of NGAL may include the proximal tubule but also increased plasma clearance. However, absolute urinary values need to be interpreted with caution due to the anti-diuretic state exerted by exercise. Normalizing uNGAL to urinary creatinine appeared to attenuate absolute rises. Given such phenomena, further work needs to be undertaken to determine the most suitable means of NGAL expression, e.g. biomarker excretion rate. Finally, given the large inter-individual response to UNGAL up-regulation, more research is needed to determine the factors responsible for large increases in some individuals and their clinical implications. These endeavours are essential if NGAL and other promising renal biomarkers are to be validated as measures of renal injury following exercise or oliguric AKI and to avoid erroneous interpretation.

\section{Conflict of Interests}

All authors do not have any conflicts-of-interest or financial disclosures to declare.

\section{Acknowledgements}

NAJ is a PhD student funded by a grant from the Betsi Cadwaladr University Health Board Charitable Funds Committee.

We would like to thank Steve Jones (Caretaker at the Treborth Athletics Track, Bangor, UK) for use of training facilities, and Dominique Mauger for her technical assistance.

\section{References}

1 Poortmans JR: Exercise and renal function. Sports Med 1984;1:125-153.

- Kachadorian WA, Johnson RE: Renal responses to various rates of exercise. J Appl Physiol 1970;28:748752.

-3 Mookerje BK, Lohr JW, Jenis EH, Heffner HM: Glomerulonephritis for the generalist. J Med 2001;32:115134.

4 Cianflocco AJ: Renal complications of exercise. Clin Sports Med 1992;11:437-451.

-5 Carter R ${ }^{3 r d}$, Cheuvront SN, Williams JO, Kolka MA, Stephenson LA, Sawka MN, Amoroso PJ: Epidemiology of hospitalizations and deaths from heat illness in soldiers. Med Sci Sports Exerc 2005;37:1338-1344.

6 Poortmans J, Dorchy H, Toussaint D: Urinary excretion of total proteins, albumin, and beta 2-microglobulin during rest and exercise in diabetic adolescents with and without retinopathy. Diabetes Care 1982;5:617623. 


\section{Kidney \\ Blood Pressure Research}

Kidney Blood Press Res 2012;36:278-289

\begin{tabular}{l|l}
\hline DOI: $10.1159 / 000343417$ & C 2012 S. Karger AG, Basel
\end{tabular}

Published online: November 28, 2012

www.karger.com/kbr

Junglee/Lemmey/Burton/Searell/Jones/Lawley/Jibani/Macdonald: NGAL Response to Exercise Proteinuria

7 Ronco C, Levin A, Warnock DG, Mehta R, Kellum JA, Shah S, Molitoris BA: Improving outcomes from acute kidney injury (AKI): Report on an initiative. Int J Artif Organs 2007;30:373-376.

8 Waikar SS, Sabbisetti VS, Bonventre JV: Normalization of urinary biomarkers to creatinine during changes in glomerular filtration rate. Kidney Int 2010;78:486-494.

9 Mishra J, Dent C, Tarabishi R, Mitsnefes MM, Ma Q, Kelly C, Ruff SM, Zahedi K, Shao M, Bean J, Mori K, Barasch J, Devarajan P: Neutrophil gelatinase-associated lipocalin (NGAL) as a biomarker for acute renal injury after cardiac surgery. Lancet 2005;365:1231-1238.

10 McCullough PA, Chinnaiyan KM, Gallagher MJ, Colar JM, Geddes T, Gold JM, Trivax JE: Changes in renal markers and acute kidney injury after marathon running. Nephrology (Carlton) 2011;16:194-199.

11 Levey AS, Stevens LA, Schmid CH, Zhang YL, Castro AF ${ }^{3 r d}$, Feldman HI, Kusek JW, Eggers P, Van Lente F, Greene T, Coresh J: A new equation to estimate glomerular filtration rate. Ann Intern Med 2009;150:604612.

12 Agarwal RP, Thanvi I, Vachhani G, Kochar DK, Rastogi A: Exercise induced proteinuria as an early indicator of diabetic nephropathy. J Assoc Physicians India 1998;469:772-774.

-13 Jefferson IG, Greene SA, Smith MA, Smith RF, Griffin NK, Baum JD: Urine albumin to creatinine ratioresponse to exercise in diabetes. Arch Dis Child 1985;60:305-310.

14 Poortmans JR, Mathieu N, De Plaen P: Influence of running different distances on renal glomerular and tubular impairment in humans. Eur J Appl Physiol Occup Physiol 1996;72:522-527.

15 Sawka MN, Burke LM, Eichner ER, Maughan RJ, Montain SJ, Stachenfeld NS: American College of Sports Medicine Position stand. Exercise and fluid replacement. Med Sci Sports Exerc 2007;39:377-390.

16 Jacobs I: Blood lactate. Implications for training and sports performance. Sports Med 1986;3:10-25.

17 Dill DB, Costill DL: Calculation of percentage changes in volumes of blood, plasma, and red cells in dehydration. J Appl Physiol 1974;37:247-248.

18 Ngal.com [homepage on the internet]. Denmark: Bioporto Diagnostics; c2012 [updated 2011; cited 2012 Mar 4]. Available from: http://ngal.com/ media/ 43917/nr2010-05-_en-_xx.pdf.

19 Quade D: Rank analysis of covariance. J Am Stat Assoc 1967;62:1187-1200.

20 Hopkins WG: A New View of Statistics. Sportscience. 2006;10:63-70.

21 Allen RA, Erickson RW, Jesaitis AJ: Identification of a human neutrophil protein of Mr 24000 that binds $\mathrm{N}$-formyl peptides: co-sedimentation with specific granules. Biochim Biophys Acta 1989;91:123-133.

22 Goetz DH, Holmes MA, Borregaard N, Bluhm ME, Raymond KN, Strong RK: The neutrophil lipocalin NGAL is a bacteriostatic agent that interferes with siderophore-mediated iron acquisition. Mol Cell 2002;10:10331043.

-23 Mishra J, Ma Q, Prada A, Mitsnefes M, Zahedi K, Yang J, Barasch J, Devarajan P: Identification of neutrophil gelatinase-associated lipocalin as a novel early urinary biomarker for ischemic renal injury. J Am Soc Nephrol 2003;14:2534-2543.

24 Mishra J, Ma Q, Kelly C, Mitsnefes M, Mori K, Barasch J, Devarajan P: Kidney NGAL is a novel early marker of acute injury following transplantation. Pediatr Nephrol 2006;21:856-863.

25 Mishra J, Mori K, Ma Q, Kelly C, Barasch J, Devarajan P: Neutrophil gelatinase-associated lipocalin: a novel early urinary biomarker for cisplatin nephrotoxicity. Am J Nephrol 2004;24:307-315.

26 Poortmans JR, Brauman H, Staroukine M, Verniory A, Decaestecker C, Leclercq R: Indirect evidence of glomerular/tubular mixed-type postexercise proteinuria in healthy humans. Am J Physiol 1988;254:F277283.

27 Poortmans JR, Blommaert E, Baptista M, De Broe ME, Nouwen EJ: Evidence of differential renal dysfunctions during exercise in men. Eur J Appl Physiol Occup Physiol 1997;76:88-89.

28 Kozlowski S, Szczepanska E, Zielinski A: The hypothalamo-hypophyseal antidiuretic system in physical exercises. Arch Int Physiol Biochim 1967;75:218-228.

29 Decavele AS, Dhondt L, De Buyzere ML, Delanghe JR: Increased urinary neutrophil gelatinase associated lipocalin in urinary tract infections and leukocyturia. Clin Chem Lab Med 2011;49:999-1003.

30 Mårtensson J, Xu S, Bell M, Martling CR, Venge P: Immunoassays distinguishing between HNL/NGAL released in urine from kidney epithelial cells and neutrophils. Clin Chim Acta 2012;413:1661-1667.

-31 Tomonaga Y, Szucs T, Ambühl P, Nock S, Risch M, Risch L: Insights on urinary NGAL obtained in a primary care setting. Clin Chim Acta 2012;413:733-739.

32 Freund BJ, Shizuru EM, Hashiro GM, Claybaugh JR: Hormonal, electrolyte, and renal responses to exercise are intensity dependent. J Appl Physiol 1991;70:900-906. 